\title{
Augmenting Reinforcement Learning with a Planning Model for Optimizing Energy Demand Response
}

\author{
Lucas Spangher \\ University of California, Berkeley \\ Berkeley, California, USA \\ Joseph Palakapilly \\ jjp126@berkeley.edu \\ University of California, Berkeley \\ Berkeley, California, USA
}

\author{
Akash Gokul \\ University of California, Berkeley \\ Berkeley, California, USA \\ akashgokul@berkeley.edu \\ Utkarsha Agwan \\ mkhattar@berkeley.edu \\ University of California, Berkeley \\ Berkeley, California, USA \\ Costas Spanos \\ University of California, Berkeley \\ Berkeley, California, USA \\ spanos@berkeley.edu
}

\author{
Manan Khattar \\ mkhattar@berkeley.edu \\ University of California, Berkeley \\ Berkeley, California, USA \\ Akaash Tawade \\ University of California, Berkeley \\ Berkeley, California, USA \\ akaasht@berkeley.edu
}

\begin{abstract}
While reinforcement learning (RL) on humans has shown incredible promise, it often suffers from a scarcity of data and few steps. In instances like these, a planning model of human behavior may greatly help. We present an experimental setup for the development and testing of an Soft Actor Critic (SAC) V2 RL architecture for several different neural architectures for planning models: an autoML optimized LSTM, an OLS, and a baseline model. We present the effects of including a planning model in agent learning within a simulation of the office, currently reporting a limited success with the LSTM.
\end{abstract}

\section{CCS CONCEPTS}

- Hardware $\rightarrow$ Smart grid; Enterprise level and data centers power issues; Impact on the environment; • Applied computing $\rightarrow$ Multicriterion optimization and decision-making.

\section{KEYWORDS}

Energy demand response, Transactive control learning, Reinforcement learning, AutoML neural architecture search, Social Energy competitions, Planning models for Reinforcement Learning

ACM Reference Format:

Lucas Spangher, Akash Gokul, Manan Khattar, Joseph Palakapilly, Utkarsha Agwan, Akaash Tawade, and Costas Spanos. 2020. Augmenting Reinforcement Learning with a Planning Model for Optimizing Energy Demand Response. In The 1st International Workshop on Reinforcement Learning for Energy Management in Buildings Cities (RLEM'20), November 17, 2020, Virtual Event, Japan. ACM, New York, NY, USA, 4 pages. https://doi.org/10. $1145 / 3427773.3427863$

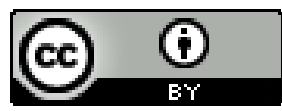

This work is licensed under a Creative Commons Attribution International 4.0 License. RLEM '2020, Nov, 2020, Yokohama, Japan

(c) 2020 Copyright held by the owner/author(s)

ACM ISBN 978-1-4503-8193-2/20/11.

https://doi.org/10.1145/3427773.3427863

\section{INTRODUCTION}

\subsection{Motivation in Energy (Background)}

The California grid (CAISO) of 2020 reported that $33 \%$ of electricity was generated from renewable sources. While admirable, this comes with challenges. Specifically, at especially sunny times in the year, system-wide generation of energy became too large for electricity grid absorb, and so CAISO simply shut down tranmission coming from solar. CAISO reported that $3-4 \%$ of its renewable energy was curtailed due to overproduction in 2019. Curtailment was spread unevenly throughout the year, with certain days in the spring reporting $20-30 \%$ curtailment. Without solutions, this problem is likely to grow non-linearly with respect to the amount of renewable energy on the grid.

\subsection{Energy storage and demand response}

One solution to curtailment is Demand Response (DR). DR entails the deferment of energy demand from when it is demanded to when it is most opportune for it to be filled. DR is basically costless, as it requires no infrastructure, so it is important as a quick and direct solution. However, consumer facing DR is nearly exclusively implemented by transmitting a coarse supply/demand price signal to consumers. Accounting for behavioral irrationalities with respect to price could dramatically improve the efficacy of this signal.

\subsection{Social Games in building energy consumptions}

The share of buildings' energy demands makes up a significant component of US energy demand and is increasing. In residential and commercial buildings, plug loads represent $30 \%$ of total electricity use ([5], [10]).

To this end, notable work has been performed in creating and administering "Social Games" - for our purposes, defined as competitions around energy use. Social Games increase the extent to which an individual is motivated to and able to save energy in their 


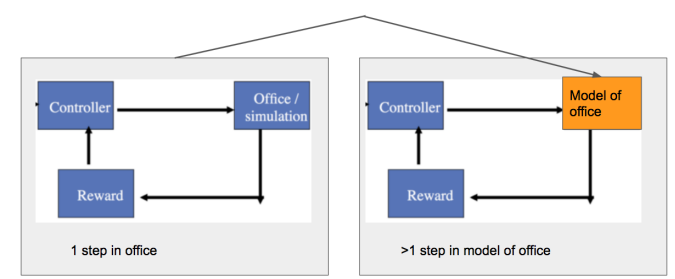

Figure 1: Diagram describing the flow of information between environment and planning model in our experiment. The agent steps once in the real world and then many times in a model of the world before alternating back.

daily functioning within their office or home ([4], [8], [6], [7]) [2], [1]).

We propose an RL agent that modifies a DR price signal within a Social Game framework. We are unaware of another study that attempts a similar outcome.

\section{METHODS}

For a quick overview on the proposed experiment/simulation, please see figure 1. In the following text, we describe each of the boxes drawn in this flow chart in detail.

\subsection{Reinforcement Learning techniques}

Reinforcement Learning (RL) as a general technique came out of the physical simulators and computer gaming - arenas that are data rich in measurements and rewards. We are dealing with a different scenario - so-called human-in-the-loop learning, where each step in the world is a day and data is very costly to attain.

As such, we need to consider off-policy RL: policies that do not rely solely on interactions with the environment, but with stored data that can be reused, with older data from other experiments, and with intelligent extrapolations. Of many architectures we explored, we present Soft Actor Critic V2 here (the "controller" in Figure 1.) We skip a description of how the architectures are structured an function; please see [9] for a tutorial.

\subsection{Planning models: improving RL agent's data efficiency}

Each step in our environment is long and costly, a feature which, when unaddressed, will hinder any algorithm from effectively learning. We propose a way to address this: planning.

Constructing a good model for human behavior in the office - and specifically response to points - could provide a way for the algorithm to "imagine" the world around it and explore more than it would otherwise. In our framework, the agent would step once in the office (i.e. deliver an action vector to the world and then observe the reward), and generate a vector of (state, action, next_state, done) that will contribute to a memory buffer specifically for office ("real world") observations. Next, stepping in the planning model space, the agent will deliver an action to the planning model, which predicts energy consumption. This tuple gets added to a separate planning memory buffer that the agent will train from We experiment with: differing the batch size of buffer sampling, whether the planning buffer has long term memory (never empties) or short term memory (empties after every step in the environment), and with a variety of planning model types.

We present four different planning models. Each model is made and trained on a simulated dataset of two months worth of energy and points data to mirror conditions of the experiment. The training dataset we used and a full explanation of the planning models we tested are detailed in [9]. Of various AutoML strategies, we present here the top performing GPyOpt LSTM search [3]; we compare this to an Oracle model (i.e., calls the same function as the simulation) an OLS and a Baseline model that returns a single value.

\subsection{Reward}

All agents use the same reward calculation. This reward is defined as the difference between the day's total cost of energy and the ideal cost of energy. The ideal cost of energy is obtained using a simple convex optimization. If $\vec{d}$ are the actual demand of energy computed for the day, $\vec{g}$ is the vector of the grid prices for the day, $E$ is the total amount of energy, and $d_{\min }, d_{\max }$ are $5 \%$ and $95 \%$ values of energy observed over the past year, then the ideal demands are calculated by optimizing the objective: $d^{*}=\min _{d} d^{T} g$ subject to the constraints $\sum_{t=0}^{10} d=E$ and $d_{\text {min }}<d<d_{\text {max }}$. Then, the reward becomes: $R(d)=\frac{d^{* T} g-d^{T} g}{d^{* T} g}$, i.e. taking the difference and scaling by the total ideal cost to normalize the outcome.

\subsection{Simulation design}

In order to test the aforementioned techniques, we will use a simulation that can provide some level of insight into the superiority of some methods over others.

2.4.1 State Space: Price generation. We include two different pricing regimes for consideration. Time of use (TOU) pricing involves is a simple, two/three-level daily price curve that changes seasonally and is declared ahead of time. We use PG\&E's price curves from 2019. Real time pricing (RTP), meanwhile, is dynamic for every hour and changes according to supply and demand in the energy market. We simulate it by subtracting the solar energy from demand of a sample building.

2.4.2 Office Workers Response. We define three deterministic responses to points, and populate our office with an equal mixture "people" who exhibit each response type. We will describe the linear response here and then refer the reader to [9] for definition of sinusoidal and threshold exponential response, as they are simple variations.

For the linear response, we define a very simple person who decreases their energy consumption linearly below a baseline with respect to points given. Therefore, if $b_{t}$ is the baseline energy consumption consumed at time $t$ and $p_{t}$ are the points given, the energy demand $d$ is $d_{t}=b_{t}-p_{t}$, clipped at $d_{\min }$ and $d_{\max }$ as defined in Section 2.3.

\section{SIMULATED RESULTS}

\subsection{Comparison of learning curves}

Here we compare the learning of agent with planning and those without: a full learning curve over $10 \mathrm{k}+$ iterations for TOU and RTP 
regimes is shown in Figure 2, whereas performance over the first thirty days, which is faithful to our upcoming experiments' constraints, is shown in figure 3. Figure 2 confirms our hypothesis that the planning models indeed help the agent learn more quickly. In Figure 3 the LSTM, labelled NN, generally outperforms the baseline model over the course of thirty days, and overperforms the OLS almost entirely. The performance of the LSTM is most pronounced when the batch size is lowest, implying that the lower the batch size, the more the agent's planning exploration is positively influenced by recent additions to buffer.

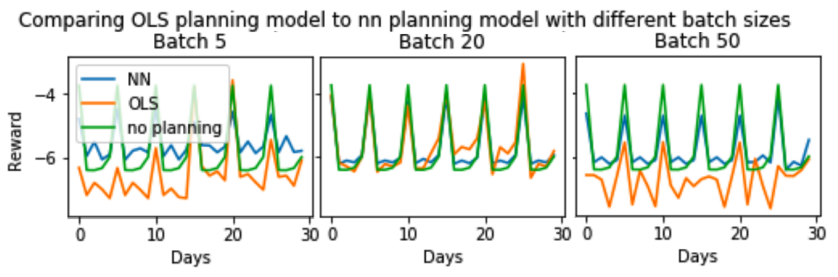

Figure 3: An exploration of the difference in batch sizes in training when comparing the LSTM planning model to the OLS planning model. This was performed in an RTP pricing regime.

\subsection{Comparison of short-term vs. long-term Buffer Memory}

Following from that finding, we found that short term memory, i.e., learning produced when the memory buffer is emptied between every step in the environment, performed better than long term memory, shown in Figure 4. The result makes sense when considering that short term memory trains with examples that are more immediately relevant to the agent's next decision.

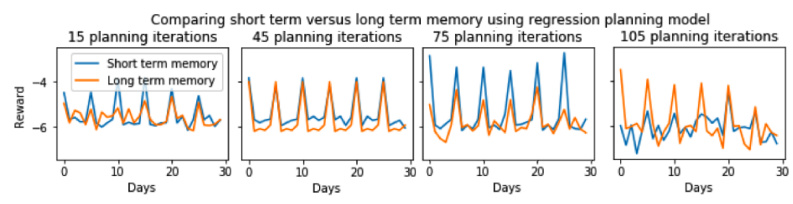

Figure 4: An exploration of the difference in memory buffer type. It is performed with the OLS because this required much lower compute than the LSTM predictions.

\section{DISCUSSION}

The results of this preliminary stop in our work are somewhat promising: we see that the machinery of the RL environment is working, and that planning models seem to have effect. While the OLS eventually helps the agent in lengthier training, it seems to hurt the RL agent's initial performance relative to the LSTM. From this, we assume that the planning model needs to be quite accurate in order to add to the simulation initially, but that when more iterations are possible, merely having a planning model present helps with training.

\section{CONCLUSION}

We have presented the results of a simulation that tests some of the components of a system we hope to test. Please see [9] for a timeline of the proposed experiment. We submit the experimental timeline and simulation for comments in this workshop, and hope to incorporate feedback as we go forward with the experiment.

\section{FUTURE WORK}

We propose strucutring the planning model further to the constraints of our problem. We envision a two part planning model that first estimates business-as-usual energy demand (i.e. without the price signal) and then corrects for a points effect; namely, an economics model taking into account elasticity of energy demand with respect to prices.

\section{ACKNOWLEDGEMENTS}

We would like to thank our wonderful and responsive peers Peter Henderson and Andreea Bobu for their thoughtful advice and commentary on the structure of the environment. We would like to also extend thanks to Alex Devonport and Adam Bouyamourn, for their comments on earlier versions of the environment. We would like to thank U.C. Berkeley for supporting us, and, finally, we would all like to thank our respective parents.

\section{REFERENCES}

[1] Ian Ayres, Sophie Raseman, and Alice Shih. 2012. Evidence from Two Large Field Experiments that Peer Comparison Feedback Can Reduce Residential Energy Usage. The fournal of Law, Economics, and Organization 29, 5 (08 2012), 992-1022. https://doi.org/10.1093/jleo/ews020 arXiv:http://oup.prod.sis.lan/jleo/article-pdf/29/5/992/2987656/ews020.pdf

[2] Ben Cowley, Jose Luiz Moutinho, Chris Bateman, and Alvaro Oliveira. 2011. Learning principles and interaction design for "Green My Place": A massively multiplayer serious game. Entertainment Computing 2, 2 (2011), 103 - 113. https://doi.org/10.1016/j.entcom.2011.01.001 Serious Games Development and Applications.

[3] Nicolas Knudde, Joachim van der Herten, Tom Dhaene, and Ivo Couckuyt. 2017. GPflowOpt: A Bayesian optimization library using tensorflow. arXiv preprint arXiv:1711.03845 (2017)

[4] I. C. Konstantakopoulos, L. J. Ratliff, M. Jin, and C. J. Spanos. 2017. Leveraging correlations in utility learning. In 2017 American Control Conference (ACC). 52495256. https://doi.org/10.23919/ACC.2017.7963770

[5] S Lanzisera, S Dawson-Haggerty, H. Y. I. Cheung, J Taneja, D Culler, and R Brown. 2013. Methods for detailed energy data collection of miscellaneous and electronic loads in a commercial office building. Building and Environment 65 (2013), 170-177.

[6] T.G. Papaioannou, N. Dimitriou, K. Vasilakis, A. Schoofs, M. Nikiforakis, F. Pursche, N. Deliyski, A. Taha, D. Kotsopoulos, C. Bardaki, S. Kotsilitis, and A. Garbi. 2018. An IoT-based gamified approach for reducing occupants' energy wastage in public buildings. Sensors (Switzerland) 18, 2 (2018). https: //doi.org/10.3390/s18020537

[7] T.G. Papaioannou and G.D. Stamoulis. 2018. Teaming and competition for demandside management in office buildings. 2017 IEEE International Conference on Smart Grid Communications, SmartGridComm 2017 2018-January (2018), 332-337. https://doi.org/10.1109/SmartGridComm.2017.8340734

[8] L. J. Ratliff, M. Jin, I. C. Konstantakopoulos, C. Spanos, and S. S. Sastry. 2014. Social game for building energy efficiency: Incentive design. In 2014 52nd Annual Allerton Conference on Communication, Control, and Computing (Allerton). 10111018. https://doi.org/10.1109/ALLERTON.2014.7028565

[9] Lucas Spangher, Akash Gokul, Manan Khattar, Joseph Palakapilly, Akaash Tawade, Adam Bouyamourn, Alex Devonport, and Costas Spanos. 2020. Prospective experiment for reinforcement learning on demand response in a social game framework. In Proceedings of the 2nd International Workshop on Applied Machine Learning for Intelligent Energy Systems (AMLIES) 2020.

[10] R. S. Srinivasan, J Lakshmanan, E Santosa, and D Srivastav. 2011. Plug load densities for energy analysis: K-12 schools,. Energy and Buildings 43 (2011), 3289 $-3294$. 
TOU Planning Comparison

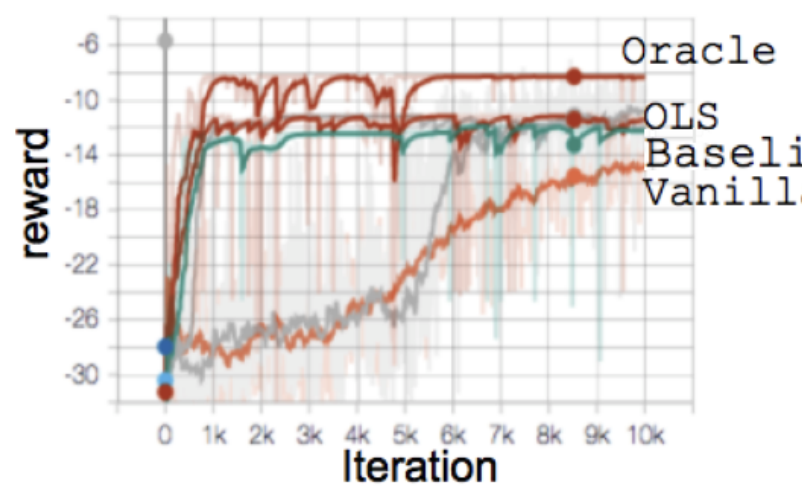

RTP Planning Comparison

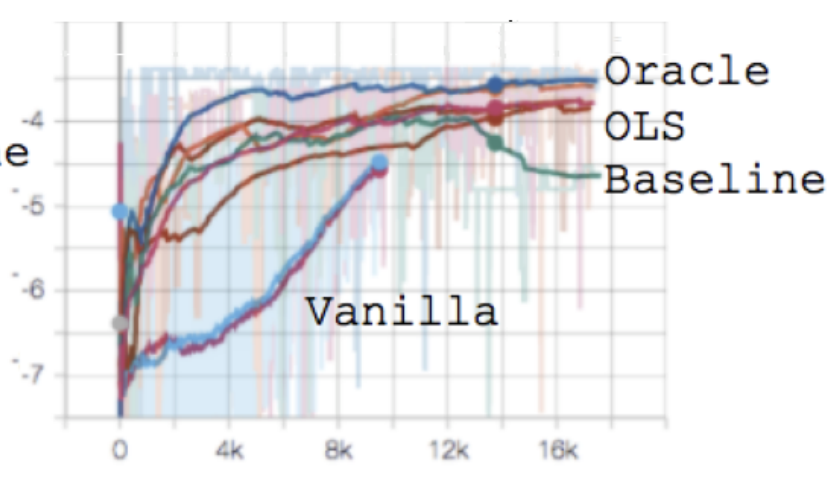

Figure 2: A figure comparing the agents with and without planning. On the left are the agents acting under a TOU price regime, and on the right are the agents acting in a RTP price regime. In both regimes, the agent's learning appears to be dramatically aided by the presence of a planning model. 